\title{
ANÁLISIS DE LAS PROPIEDADES FÍSICAS Y QUÍMICAS DE ZANAHORIA DESHIDRATADA POR ÓSMOSIS Y SECADO CONVECTIVO
}

\section{ANALYSIS OF THE PHYSICAL AND CHEMICAL PROPERTIES OF DEHYDRATED CARROT THROUGH OSMOSIS AND CONVECTIVE DRYING}

\author{
Velasco R. Edwing A. ${ }^{1}$, Salazar B. Ana M1․, Cisneros L. Olga1', Ramírez G. Luz E¹., \\ Pacheco V. Mónica M. ${ }^{1 *}$.
}

\section{RESUMEN}

En este trabajo se modificaron las características naturales de la zanahoria (Daucus carota L.) aplicando de forma consecutiva procesos de deshidratación osmótica y secado por aire caliente. En particular, se estudió el efecto de una solución hipertónica de sacarosa concentrada a 50 $60 \% \mathrm{p} / \mathrm{p}$ y una temperatura de secado de $60^{\circ} \mathrm{C}$, sobre las propiedades físicas y químicas del vegetal. Las muestras deshidratadas se analizaron comparativamente frente al alimento en fresco mediante la determinación de la humedad, actividad de agua, sólidos solubles, $\mathrm{pH}$, porcentaje de acidez, color y resistencia a la compresión. Se encontró que el proceso de deshidratación combinado promueve una reducción en el peso de la zanahoria mayor al $70 \%$, actividad de agua por debajo de 0,4, acidez 
cercana al $2 \%$ y sólidos solubles entre 28 y $35{ }^{\circ} \mathrm{Bx}$, sin modificar significativamente el color natural del alimento.

*Autor a quien debe dirigirse la correspondencia. Pacheco V. Mónica M.*. Correo electrónico: *monica.pacheco@unipaz.edu.co.
Palabras clave: Deshidratación, Solución hipertónica, Aire caliente, Vegetal.

\begin{abstract}
In this work the natural characteristics of the carrot (Daucus carota $L$.) were modified by consecutively applying osmotic dehydration and convective drying processes. In particular, it was studied both the effect of a hypertonic solution of concentrated sucrose at $50-60 \% \mathrm{w} / \mathrm{w}$ and a drying temperature of $60{ }^{\circ} \mathrm{C}$, on the physical and chemical properties of vegetable slices. The dehydrated samples were analyzed comparatively to the fresh food by determining the moisture, water activity, soluble solids, $\mathrm{pH}$, acidity and compressive strength. It was found that the combined process allows a reduction in carrot weight greater than $70 \%$, water activity below 0,4 , acidity close to $2 \%$, soluble solids between 28 and $35{ }^{\circ} \mathrm{Bx}$, without significantly changing the natural color of the food.
\end{abstract}

KEYWORDS: Dehydration, Hypertonic Solution, Hot Air, Vegetable.

\section{INTRODUCCIÓN}

La zanahoria es una de las hortalizas de raíz comestible más cultivadas del planeta, está compuesta principalmente por agua (86\%), carbohidratos (10,6\%), proteínas $(0,9 \%)$, grasa $(0,2 \%)$, fibra cruda $(1,2 \%)$, carotenos $(5,33 \mathrm{mg} / 100 \mathrm{~g})$, vitamina C (4 $\mathrm{mg} / 100 \mathrm{~g})$, Ca $(80 \mathrm{mg} / 100 \mathrm{~g})$, Fe $(2,2$ $\mathrm{mg} / 100 \mathrm{~g})$ y $P(53 \mathrm{mg} / 100 \mathrm{~g})$, entre otras vitaminas y minerales (Arscott y Tanumihardjo, 2010 y Sharma et al., 2012). Gracias a la gran variedad de nutrientes que posee, la zanahoria es considerada como un alimento ideal en la dieta de los seres humanos, así como también, un vegetal con alto potencial a nivel medicinal, especialmente para el tratamiento de enfermedades oculares y el cáncer. Preferentemente, se utiliza en la preparación de sopas, guisos, curry, dulces, jugos, encurtidos fermentados y ensaladas, no obstante, la elaboración este tipo de productos a partir de la zanahoria 
se ha visto limitada debido a su relativa corta vida útil (3 - 4 días); tal desventaja está asociada con el alto contenido de agua, el cual promueve el deterioro fisicoquímico del vegetal y proliferación microbiana (Singh et al., 2013, VegaMercado et al., 2001 y Lewicki, 2006).

Una estrategia para alargar la vida de este tipo de alimentos perecederos es el uso de tratamientos que disminuyan el contenido de agua (Chou, S.K. y Chua, 2001, Nakagawa, K. y Ochiai, 2015 y Ahmed, 2016). El secado convectivo es uno de los métodos de deshidratación más utilizados por su simplicidad y facilidad operacional a nivel industrial, y se basa en un proceso simultáneo de transferencia de masa y calor acompañado por un cambio de fase del agua; generalmente se usa aire caliente para remover la humedad del tejido vegetal. De acuerdo con Mihoubi et al. (2009), este proceso logra disminuir el peso del alimento hasta en un $90 \%$, condición que permite su conservación por largos periodos. En contraste con estos aspectos, el secado con aire caliente también es conocido porque altera las propiedades sensoriales del alimento, además de acarrear un elevado costo energético.

La deshidratación osmótica es otra técnica utilizada para disminuir el contenido de agua de frutas y vegetales. Consiste en sumergir la matriz biológica en una solución acuosa hipertónica de sales, azúcares y/o alcoholes, con el fin de promover la deshidratación gracias al gradiente de presión osmótica. En este proceso, la pared celular del alimento actúa como una membrana semipermeable que permite el paso del agua desde el alimento hasta la solución hipertónica (Torregginni, 1993) y en algunos casos de vitaminas y pigmentos solubles; así mismo, de forma simultánea el alimento es impregnado con solutos procedentes de la solución hipertónica (Dixon y Jen, 1977 y Lerici et al., 1985). La principal desventaja de esta técnica frente a otros procesos de deshidratación como el secado, es que no reduce el contenido de agua del alimento hasta niveles que inhiban el crecimiento bacteriano.

Varios autores han reportado que el uso combinado de los tratamientos de ósmosis y secado con aire caliente, tiene mejores resultados que utilizándolos de forma individual, ya que permite alcanzar niveles altos de deshidratación, mejora las características funcionales, sensoriales $y$ nutricionales del alimento, y disminuye el costo asociado al proceso [Revaskar et al., 2007 y Vázquez-Vila et al., 2009). Según Raoult-Wack (1994), las matrices vegetales que se someten a este tratamiento combinado presentan mejor color y sabor que las que solo son tratadas con secado, debido a que tienen menor tiempo de exposición a altas temperaturas. Se ha reportado que las variables más relevantes de los procesos mencionados 
son la concentración de la solución osmótica y el tiempo y las temperaturas del secado [Della Rocca et al., 2013, VegaMercado et al., 2001, Nanjundaswamy et al., 1978). Estudios realizados por Torres et al., (2007) encontraron que el cambio en la concentración de la solución hipertónica de sacarosa afectaba directamente la humedad de muestras de papaya. Estos autores concluyeron que el gradiente de presión osmótica tiene mayor influencia sobre la transferencia efectiva de masa, que la temperatura del proceso. Así mismo, Sutar y Prasad et al., (2011) reportaron que tanto la deshidratación como la impregnación de solutos aumentan a medida que se incrementan la concentración de la solución osmótica y la temperatura del proceso.

En consonancia con los aportes realizados por los autores citados, en este trabajo se estudia el efecto combinado del tratamiento osmótico y el secado convectivo sobre las propiedades físicas y químicas de la zanahoria, analizando específicamente la influencia de la concentración de la solución hipertónica y el tiempo del proceso.

\section{MATERIALES Y MÉTODOS}

\section{Preparación de las muestras}

Se utilizaron zanahorias frescas en óptimo estado de maduración y libres de magulladuras y picaduras, con un peso promedio de $200 \mathrm{~g}$, longitud de $15 \mathrm{~cm}$ y diámetro de la base del cono de $6 \mathrm{~cm}$; se adquirieron en mayo de 2016 en un supermercado local en Barrancabermeja, Santander, Colombia. Las zanahorias fueron lavadas, peladas y cortadas en rodajas de $0,5 \mathrm{~mm}$ de espesor utilizando una tajadora eléctrica (JAVAR, modelo GE220 1F). Se escogieron las rodajas con un diámetro aproximado de $3 \mathrm{~cm}$ con el fin de asegurar una similitud en el área superficial de cada muestra.

\section{Deshidratación osmótica y secado}

El proceso de osmodeshidratación se realizó por inmersión de las muestras de zanahoria en una solución de sacarosa comercial (pureza de $99 \%$ ) concentrada a 50,55 y $60 \%$ p/p durante un periodo de 2$6 \mathrm{~h}$. La temperatura y agitación durante el proceso osmótico se mantuvieron constantes a $100 \mathrm{rpm}$ y $40 \stackrel{\circ}{\circ}$, respectivamente, utilizando una incubadora de agitación orbital (LABNET, modelo 15311-DS). En este proceso se utilizó una relación de $1 \mathrm{~g}$ de zanahoria por $1 \mathrm{~mL}$ de solución de sacarosa. Finalizado el tiempo de inmersión, las rodajas de zanahoria se retiraron de la solución y se lavaron con agua para remover residuos de sacarosa adheridos a la superficie. Inmediatamente después, las rodajas se secaron con papel adsorbente con el fin de 
retirar el agua libre. Posterior al tratamiento de osmodeshidratación, las muestras de alimento fueron sometidas a un proceso de secado a $60 \stackrel{\circ}{\circ}$ en un horno con convección forzada (BINDER, modelo FD 23) durante un periodo de 3-9 h.

\section{Extracción del zumo de zanahoria}

Las rodajas de zanahoria fresca $y$ deshidratada fueron maceradas en un mortero de porcelana durante $5 \mathrm{~min}$ a temperatura ambiente. El producto macerado fue centrifugado a $3000 \mathrm{rpm}$ en una centrifuga (Labnet, Spectrafuge 6C) durante $5 \mathrm{~min}$; de la mezcla resultante se separó el zumo por decantación y se descartó el pellet de biomasa restante.

\section{Caracterizaciones}

El contenido de humedad se determinó mediante el seguimiento de la pérdida de peso por desecación en horno con convección forzada (BINDER, modelo FD 23) a $125^{\circ} \mathrm{C}$ durante $4 \mathrm{~h}$, de acuerdo con Bradley (2010). La actividad de agua (Aw) se evaluó con un medidor portátil (ROTRONIC, modelo HP23-AW). Los sólidos solubles totales se hallaron en términos de ${ }^{\circ}$ Brix a $20{ }^{\circ} \mathrm{C}$ utilizando un refractómetro portátil (Zhifong, modelo FG109). El pH se midió a $20 \stackrel{\circ}{\mathrm{C}}$ usando un potenciómetro (OAKTON, modelo pH 510) y la acidez se calculó en términos de ácido cítrico (g ácido cítrico/ $100 \mathrm{~g}$ de alimento) mediante titulación con una solución $0,1 \mathrm{~N}$ de $\mathrm{NaOH}$ (Merck, pureza de $99 \%$ ), ambos conforme a la metodología reportada por Sadler y Murphy (2010). La humedad y el Aw se determinaron utilizando las rodajas de zanahoria mientras que las demás caracterizaciones se realizaron usando el zumo del alimento.

El color se determinó por evaluación visual utilizando como sistema de referencia la tabla cromática de colores (Sanz y Gallego, 2001). La tonalidad del color se analizó utilizando el software libre ImageJ en el modo RGB (Pascau y Mateos, 2013). La resistencia a la compresión se determinó utilizando un analizador de textura (STABLE MICRO SYSTEMS, TA-XT2i) con una celda de carga de $50 \mathrm{~kg}_{f}$ y un embolo de acrílico de diámetro 41,1 mm. Para éste análisis $8,4 \mathrm{~g}$ de zanahoria deshidratada fueron cargados en un recipiente cilíndrico de acrílico (Diámetro interno $=49,9 \mathrm{~mm}$ y altura $=68,3 \mathrm{~mm}$ ) alcanzando una altura inicial de aproximadamente $20 \mathrm{~mm}, y$ comprimidos hasta la mitad de su altura (deformación del 50 \%). Las velocidades del análisis fueron: pre-test $=2 \mathrm{~mm} / \mathrm{s}$, test $=1 \mathrm{~mm} / \mathrm{s}$ y post-test $=2 \mathrm{~mm} / \mathrm{s}$.

\section{RESULTADOS Y DISCUSIÓN}

\section{Efecto del proceso de deshidratación osmótica}

En la Figura 1 se muestran los resultados de la variación del peso y sólidos solubles de las muestras de zanahoria 
deshidratadas. En general se observa que el tratamiento osmótico reduce el peso de las muestras de zanahoria entre 30-40\%, mientras que aumenta entre 5 y 6 veces su contenido de sólidos solubles. El peso disminuye proporcionalmente con el aumento en la concentración de la solución de sacarosa, en tanto que los sólidos solubles sufren incrementos asociados a la utilización de soluciones de sacarosa entre 50 y $55 \%$ p/p; después de lo cual no se observan variaciones importantes. La disminución en el peso indica que ocurrió la liberación de agua de la matriz del alimento, mientras que el aumento en los sólidos solubles da a entender que hubo deposición de solutos en las muestras, lo cual es coherente con lo reportado por Singh et al. (2010), Pacheco-Angulo et al. (2016) y Simal et al. (1998). De acuerdo con Rastogi et al. (2014) la deshidratación es causada por la diferencia en los potenciales químicos entre el agua y la solución hipertónica (que para este trabajo es una dilución de sacarosa), que da lugar a un flujo de varios componentes: el agua sale del alimento y los solutos entran, principalmente en las primeras 2 - $3 \mathrm{~h}$ de inmersión. Después de este tiempo la diferencia en el contenido de agua en el alimento y en la solución disminuye gradualmente hasta que el sistema alcanza un estado de equilibrio dinámico. Al observar la Figura 1 se encuentra que el tiempo de inmersión donde ocurre mayor deshidratación también está entre las primeras 2-4 h, similar a lo mencionado anteriormente.

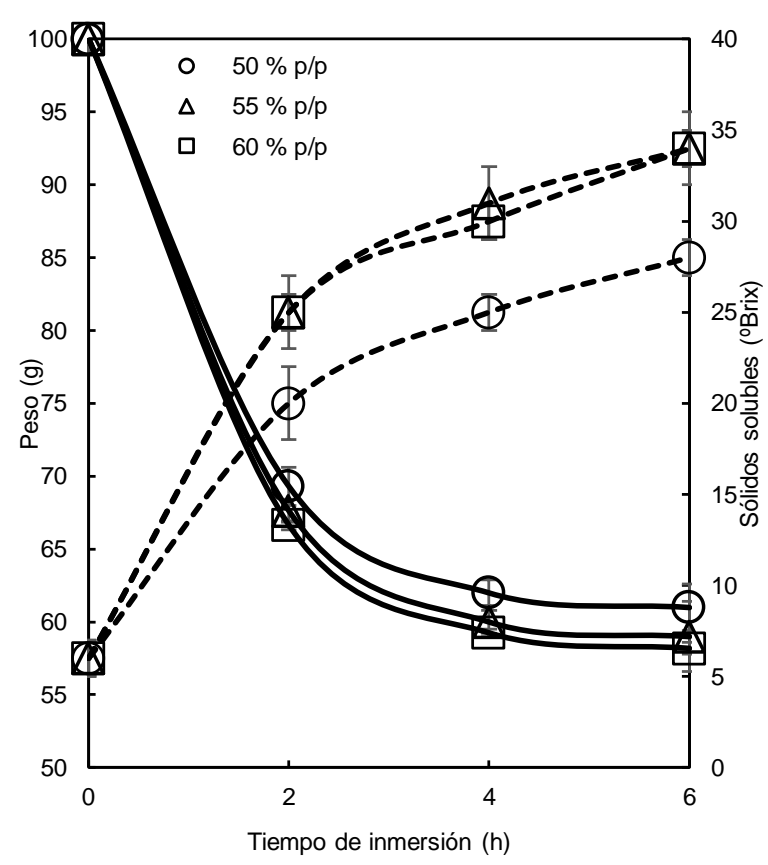

Figura 1. Comportamiento del peso (líneas continuas) y los sólidos solubles (líneas discontinuas) de las muestras de zanahoria deshidratadas utilizando soluciones de sacarosa concentradas a 50,55 y $60 \%$ p/p.

Los resultados son el promedio de seis réplicas y las barras de error representan la desviación estándar. En la Figura 2 se presentan los resultados de la variación del $\mathrm{pH}$ y el porcentaje de acidez de las muestras de zanahoria osmodeshidratadas en función del tiempo del proceso, utilizando varias concentraciones de la solución de sacarosa. Se observa que al incrementar el tiempo de deshidratación ocurre tanto un incremento en el pH como una disminución en la acidez. Sin embargo, tales comportamientos se presentan antes de las $4 \mathrm{~h}$ de tratamiento; después de este 
tiempo tanto el $\mathrm{pH}$ como la acidez tienden a estabilizarse. Así mismo, se observa que al aumentar la concentración de sacarosa sube el $\mathrm{pH}$, y como es de esperarse, baja el porcentaje de acidez. De acuerdo con estos resultados se puede presumir que durante la osmodeshidratación el agua que salió del alimento arrastró consigo ácidos orgánicos y en efecto cambió la acidez del alimento; esto se corrobora al observar que los tiempos en que se estabiliza el peso (Figura 1) y la acidez (Figura 2) son similares. En coherencia con lo expuesto, Raoult-Wack (1994) menciona que el proceso de deshidratación osmótica incrementa la relación entre los contenidos de sacarosa/ácidos en el alimento.

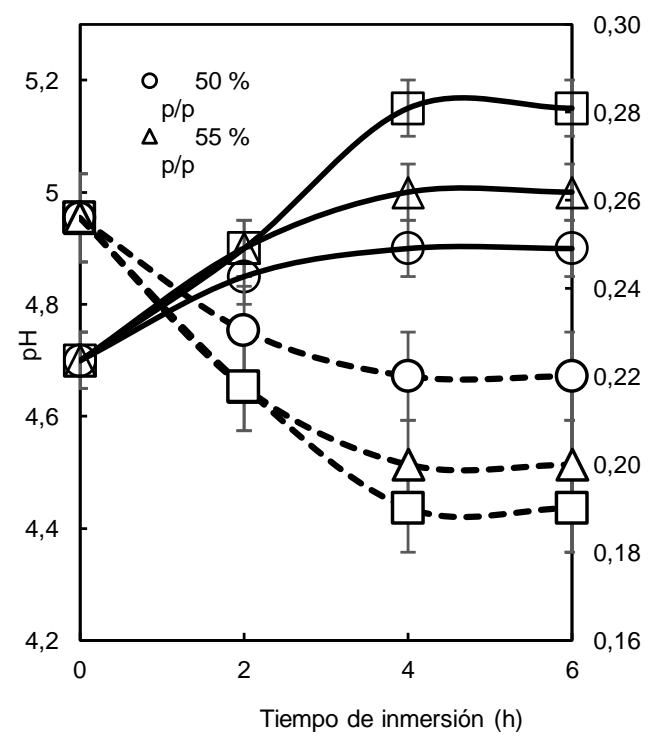

Figura 2. Comportamiento del pH (líneas continuas) y de la acidez (líneas discontinuas) de las muestras de zanahoria osmodeshidratadas utilizando soluciones de sacarosa concentradas a 50,55 y $60 \%$ $\mathrm{p} / \mathrm{p}$.
Los resultados son el promedio de seis réplicas y las barras de error representan la desviación estándar.

\section{Efecto del proceso de secado}

Como se señaló anteriormente, el proceso de deshidratación osmótica promovió una reducción en el peso de la zanahoria del 40 $\%$, debido principalmente a la salida de agua del alimento. No obstante, se estima que la zanahoria tiene un contenido de agua mayor al $80 \%$, lo que indica que el tratamiento no eliminó la humedad total. Como alternativa para mejorar la deshidratación, se realizó un proceso posterior de secado. Los resultados de la variación del peso y la actividad de agua durante este secado se muestran en la Figura 3. Se observa que ambas variables disminuyen con el tiempo de tratamiento, no obstante, la mayor parte de esta variación (36-53 \% del peso y $60 \%$ de la actividad de agua) ocurrió en las primeras 3 horas del secado. La similitud en el comportamiento del peso y la actividad de agua indican, tal como se esperaba, que los cambios observados en la Figura 3 ocurrieron por la pérdida de agua. La aparente estabilización en el peso después de $3 \mathrm{~h}$ mostrada en la Figura 3 también fue reportada por Kowalski et al., (2013). Esto indica que el tiempo de secado para este tipo de muestras no debe superar las $3 \mathrm{~h}$.

Por otra parte, al verificar el efecto del tratamiento de osmosis sobre el secado, se observa que a mayor concentración de la 
solución de sacarosa es menor la pérdida de peso durante el secado. La influencia de la concentración de la solución osmótica sobre la variación del peso puede estar asociada con la deposición de sacarosa en la matriz del alimento. Tal deposición pudo provocar una disminución en el coeficiente de difusión del agua en la interfase alimento-aire, así como lo explica Rastogi et al., (2014), y en efecto resistir la salida del agua.

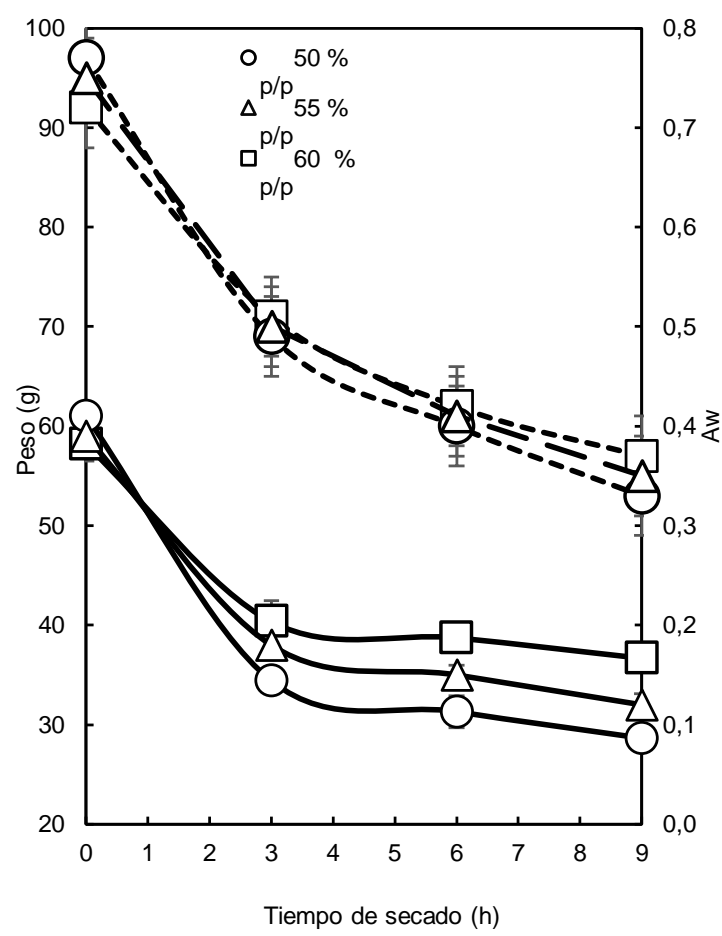

Figura 3. Comportamiento del Peso (líneas continuas) y de la Actividad acuosa (líneas discontinuas) de las muestras de zanahoria osmodeshidratadas, durante el proceso de secado a $60 \stackrel{\circ}{ } \mathrm{C}$.

Los resultados son el promedio de seis réplicas y las barras de error representan la desviación estándar. En la Figura 4 se presentan los resultados de la prueba de resistencia a la compresión de las muestras de zanahoria deshidratadas por ósmosis y luego por secado con aire caliente. Se observa que al utilizar una solución osmótica más concentrada disminuye la resistencia a la compresión de las muestras de zanahoria, así mismo, presentan mayor deformación. Al comparar estos resultados con los presentados en la Figura 1 se puede inferir que están relacionados inversamente con la deposición de solutos. Teniendo en cuenta esto, es posible que el exceso de sacarosa depositada en la superficie de la zanahoria haya cristalizado durante la etapa de secado, atribuyéndole a la muestra rigidez y fragilidad (crocancia), lo que disminuye la resistencia a la compresión. Tales características son reconocidas en alimentos tipo snack.

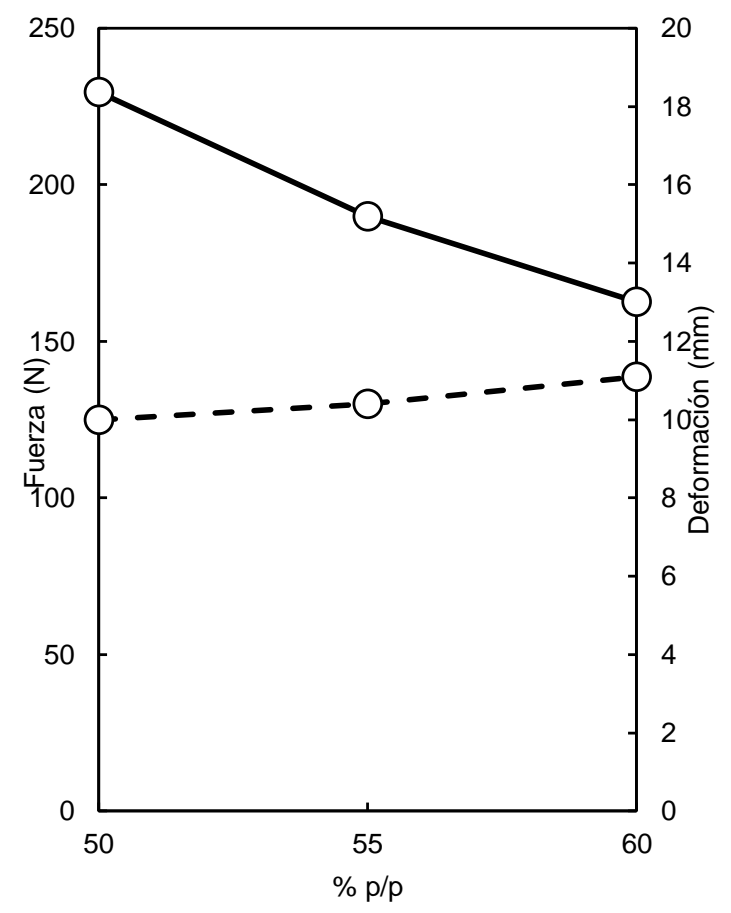


Figura 4. Resultados de la Fuerza aplicada sobre las muestras de zanahoria deshidratada (líneas continuas) y la deformación causada (líneas discontinuas) durante la prueba de compresión en función de la concentración de la solución osmótica.

La variación en el color como una función del tratamiento de las muestras de zanahoria también fue estudiada. Los procesos simultáneos de osmodeshidratación y secado convectivo no alteraron significativamente el color de la zanahoria. Según la escala monocromática de colores (Sanz y Gallego, 2001), las muestras de zanahoria fresca y deshidratada presentaron un color naranja. No obstante, el incremento en la concentración de la solución osmótica generó un leve oscurecimiento en el alimento. En la Figura 5 se muestran los histogramas de color de las muestras de zanahoria fresca y tratada; donde la distribución del color de claro a oscuro se observa de derecha a izquierda. Al comparar las imágenes de la Figura 5 (a-d) se observa que el incremento en la concentración de la solución osmótica causa un corrimiento en la distribución del color hacia la izquierda, lo que demuestra que el tratamiento causó el oscurecimiento de la zanahoria. Este cambio en el color pudo ser provocado por la deposición de solutos y la pérdida de ácidos orgánicos de la matriz del alimento, discutido previamente. La preservación de la tonalidad naranja, a pesar del oscurecimiento del alimento, indica que no hubo modificaciones considerables en el contenido de carotenos (Kowalski et al., 2013). Resultados similares fueron reportados por Torregginni et al. (1993). Tal como lo mencionan Rastogi et al., (2014) y Singh et al., (2010), la preservación del color se debe a que las bajas temperaturas y la liberación de agua sin cambio de estado durante la osmodeshidratación no causan una pérdida de pigmentos considerable.

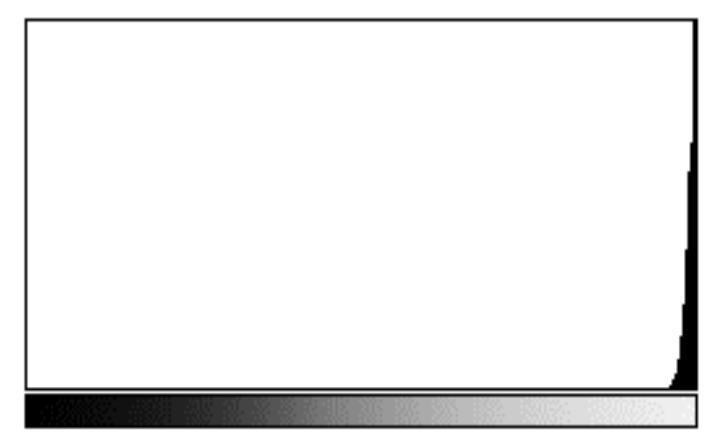

(a)

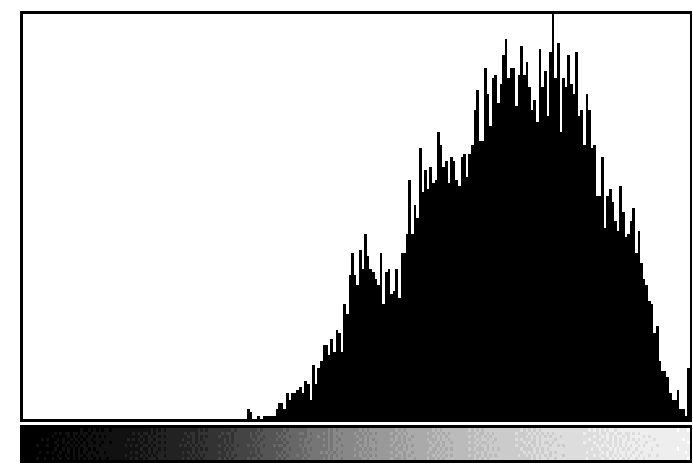

(b)

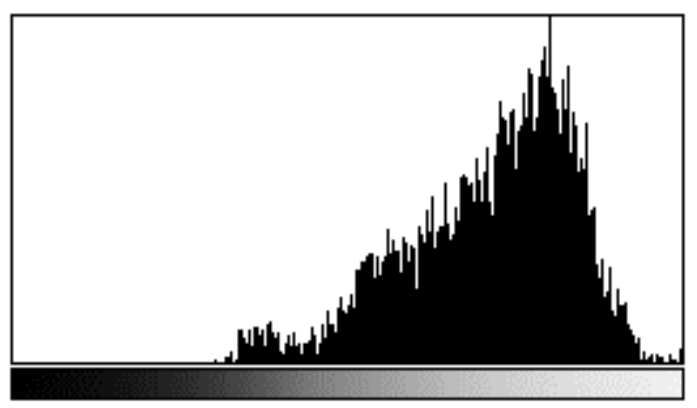

(c) 


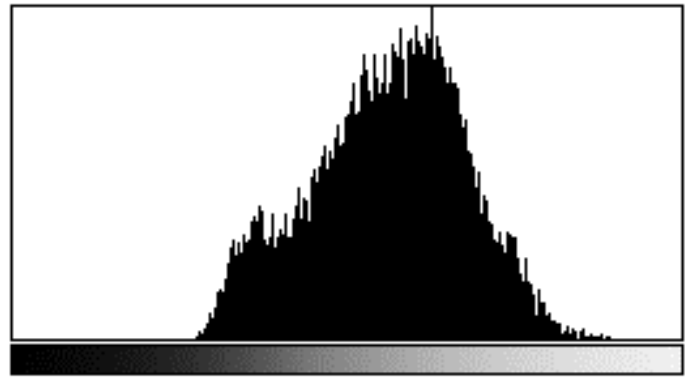

(d)

\section{CONCLUSIONES}

El proceso combinado de deshidratación osmótica y secado convectivo permite la obtención de muestras de zanahoria con una reducción en el peso mayor al $70 \%$, actividad de agua por debajo de 0,4 , acidez cercana al $2 \%$ y sólidos solubles entre 28 y $35^{\circ} \mathrm{Bx}$, que mantienen su color naranja.

Individualmente, la deshidratación osmótica utilizando soluciones de sacarosa concentradas a $50-60 \% \mathrm{p} / \mathrm{p}$, reduce el peso de la zanahoria entre el 30 y $40 \%$, así como también promueve la deposición de solutos en el alimento.
Figura 5. Histograma de color de las zanahorias fresca (a) y deshidratada por ósmosis y secado convectivo utilizando soluciones de sacarosa a $50 \%$ p/p (b), 55 $\% p / p$ (c) y $60 \% p / p$ (d).
La mayor relación disminución de peso/solutos depositados se alcanzó con la solución a 50 \% p/p.

La pérdida de agua en la ósmosis ocurre predominantemente en las primeras $4 \mathrm{~h}$ y en el secado en las primeras $2 \mathrm{~h}$, mientras que la deposición de solutos predomina en los periodos posteriores.

A las condiciones establecidas en este trabajo, el proceso de deshidratación modifica sustancialmente la humedad del alimento pero no cambia significativamente sus propiedades sensoriales.

\section{REFERENCIAS BIBLIOGRÁFICAS}

Ahmed, I., Qazi, I.M. y Jamal, S. Developments in osmotic dehydration technique for the preservation of fruits and vegetables. (2016). Innovative Food Science and Emerging Technologies. 34: 29-43.

Arscott, S.A. And Tanumihardjo, S.A. Carrots of many colors provide basic nutrition and bioavailable phytochemicals acting as a functional food. (2010).
Comprehensive Reviews in Food Science and Food Safety. 9: 223-239.

Bradley, R.J. Food Analysis. USA: Springer Science+Business Media, 2010. p. 85107.

Chou, S.K. y Chua, K.J. New hybrid drying technologies for heat sensitive foodstuffs. (2001). Trends in Food Science \& Technology. 12(10): 359-369.

Della Rocca, P.A., Roche, L.A. y Mascheroni, R.H. Estudio de la 
@)LIMENTECH CIENCIA Y TECNOLOGÍA ALIMENTARIA ISSN 1692-7125. Volumen 14 No. 2, p. 42 - 53, año 2016 Facultad de Ingenierías y Arquitectura

Universidad de Pamplona

transferencia de agua durante la deshidratación osmótica de zanahoria. (2013). Proyecciones. 11(2): 81-91.

Dixon, G.M. y Jen, J.J. Changes of sugar and acid in osmovac dried apple slices. (1977). Journal of Food Science. 42: 1126-1131.

Kowalski, S.J., Szadnziska, J. y Łechtanska, J. Non-stationary drying of carrot: Effect on product quality. (2013). Journal of Food Engineering. 118: 393399.

Lerici, C.L., Pinnavaia, G., Dalla-Rosa, M. y Bartolucci, L. Osmotic dehydration of fruit: influence of osmotic agents on drying behaviour and product. (1985). Journal of Food Science. 50: 1217-1219.

Lewicki, P.P. Design of hot air drying for better foods. (2006). Trends in Food Science \& Technology. 17(4): 153-163.

Mihoubi, D., Timoumi, S. y Zagrouba, F. Modelling of convective drying of carrot slices with IR heat source. (2009). Chemical Engineering and Processing. 48: 808-815.

Nakagawa, K. y Ochiai, T. A mathematical model of multi-dimensional freeze-drying for food products. (2015). Journal of Food Engineering. 161: 55-67.

Nanjundaswamy, A.M., Radhakrishnaiah, S.G., Balachandran, C., Saroja, S. y Murthy, R.K. Studies on development of new categories of dehydrated products from indigenous fruits. (1978). Indian Food Packer. 22: 91-93.

Pacheco-Angulo, H., Herman-Lara, E., García-Alvarado, M.A. And Ruiz-López, I.I. Mass transfer modeling in osmotic dehydration: Equilibrium characteristics and process dynamics under variable solution concentration and convective boundary. (2016). Food and Bioproducts Processing. 97: 88-99.

Pascau. J. And Mateos, J. Image processing with ImageJ. (2013). Disponible en: http://imagej.nih.gov/ij/docs/index.html

Pua, R. Amparo, Barreto, G. R, y Ariza C. S. (2015). Extracción y caracterización de la pectina obtenida a partir de la cáscara de limón Tahití (citrus $x$ latifolia) en dos estados de maduración. Revista @limentech, Ciencia y Tecnología Alimentaria. ISSN 1692-7125. Volumen $13, N^{\circ} 2, p 5-16$.

Raoult-Wack, A.L. Advances in osmotic dehydration. (1994). Trends in Food Science \& Technology. 5: 255-260.

Rastogi, N.K., Raghavarao, K. y Niranjan, K. Chapter 11: Recent Developments in Osmotic Dehydration. (2014). Emerging Technologies for Food Processing. 181212.

Revaskar, V., Sharma, G.P., Verma, R.C., Jain, S.K. And Chahar, V.K. Drying 
@LIMENTECH CIENCIA Y TECNOLOGÍA ALIMENTARIA ISSN 1692-7125. Volumen 14 No. 2, p. 42 - 53, año 2016 Facultad de Ingenierías y Arquitectura

Universidad de Pamplona

behaviour y energy requirement for dehydration of white onion slices. (2007). International Journal of Food Engineering. 3(5): 1-16.

Sadler, G.D. y Murphy, P.A. Food Analysis. USA: Springer Science+Business Media, 2010. p. 219-230.

Sanz, J.C. y Gallego, R. Diccionario Akal del Color. Akal, 2001, p. 600-650.

Sharma, K.D., Karki, S., Thakur, N.S. y Attri, S. Chemical composition, functional properties and processing of carrot - a review. (2012). Journal of Food Science and Technology. 49(1): 22-32.

Simal, S., Benedito, J., Sanchez, E.S. y Rossello, C. Use of ultrasound to increase ass transport rates during osmotic dehydration. (1998). Journal of Food Engineering. 36: 323-336.

Singh, B., Panesar, P.S., Nanda, V. y Kennedy, J.F. Optimization of osmotic dehydration process of carrot cubes in mixtures of sucrose and sodium chloride solutions. (2010). Food Chemistry. 123: 590-600.

Singh, C., Sharma, H.K. y Sarkar, B.C. (2010). Influence of process conditions on the mass transfer during osmotic dehydration of coated pineapple samples. Journal of Food Processing and Preservation. 34: 700-714.
Singh, P., Kulshrestha, K. y Kumar, S. Effect of storage on $\beta$-carotene content and microbial quality of dehydrated carrot products. (2013). Food Bioscience. 2: 3945.

Sutar, P.P. y Prasad, S. Optimization of osmotic dehydration of carrots under atmospheric and pulsed microwave vacuum conditions. (2011). Drying Technology. 29: 371-380.

Torregginni, D. Osmotic dehydration in fruits and vegetable processing. (1993). Food Research International. 26: 59-68.

Torres, J.D., Talens, P., Carot, J.M., Chiralt, A. y Escriche, I. Volatile profile of mango (Mangifera indica L.), as affected by osmotic dehydration. (2007). Food Chemistry. 101: 219-228.

Vázquez-Vila, M.J., Chenlo-Romero, F., Moreira-Martínez, R. y Pacios-Penelas, B. Dehydration kinetics of carrots (Daucus carota L.) in osmotic and air convective drying processes. (2009).

Spanish Journal of Agricultural Research. 7(4): 869-875.Vega-Mercado, H., AngoraNieto, M.M. y Bartosa-Cánovas, G.V. (2001). Advanced in dehydration of food. Journal of Food Engineering. 49: 271-89.

Vega-Mercado, H., Angora-Nieto, M.M. y Bartosa-Cánovas, G.V. Advanced in dehydration of food. (2001). Journal of Food Engineering. 49: 271-289. 